Pacific Journal of Mathematic 


\title{
QUASI-AFFINE TRANSFORMS OF SUBNORMAL OPERATORS
}

\author{
Che-Kao Fong
}

For an operator $T$ which is a quasi-affine transform of a subnormal operator $S$, we show that: (1) if $S^{*}$ has no point spectrum and $f: \lambda \mapsto(T-\lambda)^{-1} x$ is defined on an open set $\Omega$, then there is a dense subset $\Omega_{0}$ of $\Omega$ such that $f \mid \Omega_{0}$ is analytic; and (2) if $\Sigma$ is a spectral set of $T$ and $Q$ is a peak set of $R(\Sigma)$, then the spectral manifold $X_{T}(Q)$ is a reducing subspace of $T$ and $Q$ is a spectral set of $T \mid X_{T}(Q)$.

1. Introduction. We generalize results of Putnam [5] and [6] which concern local spectral properties of subnormal operators to quasi-affine transforms of subnormal operators.

Before we proceed, we fix some notation and terminology. All operators are assumed to be linear, bounded and defined on Hilbert spaces. For an operator $T$, we write $\sigma(T)$ for the spectrum of $T$. For an operator $T$ defined on $\mathscr{H}$ and a closed set $F$ in the complex plane $\mathbf{C}$, we write $\mathscr{X}_{T}(F)$ for those $x$ in $\mathscr{H}$ such that there exists a vector-valued analytic function $f$ from $\mathbf{C} \backslash F$ into $\mathscr{H}$ satisfying $(T-\lambda) f(\lambda)=x$ for all $\lambda \in \mathbf{C} \backslash F$. An operator $T$ has the single-valued extension property if whenever $g$ is a vector-valued analytic function defined on an open set in C with $(T-\lambda) g(\lambda) \equiv 0$ then $g(\lambda) \equiv 0$. (See Colojoară and Foiaş [1].) By a quasi-affinity we mean a (bounded linear) mapping $W: \mathscr{H} \rightarrow \mathscr{K}$ between two Hilbert spaces $\mathscr{H}$ and $\mathscr{K}$ which is one-one and has its range dense in $\mathscr{K}$. An operator $T$ defined on $\mathscr{H}$ is said to be a quasi-affine transform of an operator $S$ defined on $\mathscr{K}$ if there is a quasi-affinity $W: \mathscr{H} \rightarrow \mathscr{K}$ such that $S W=W T$.

Suppose we have $N W_{0}=W_{0} T$, where $N$ is a normal operator defined on $\mathscr{K}_{0}, T$ is an operator on $\mathscr{H}$ and $W_{0}: \mathscr{H} \rightarrow \mathscr{K}_{0}$ is one-one. Let $\mathscr{K}$ be the closure of the range of $W_{0}$ and $W: \mathscr{H} \rightarrow \mathscr{K}$ be the map which has the same value as $W_{0}$ at each point in $\mathscr{H}$. Then $\mathscr{K}$ is invariant under $N$ and $S W=W T$ where $S$ is the subnormal operator defined by restricting $N$ to $\mathscr{K}$. Therefore $T$ is a quasi-affine transform of a subnormal operator. Conversely, suppose $T$ is a quasi-affine transform oof a subnormal operator $S$. Let $W$ be a quasi-affinity such that $S W=W T$ and $N$ be a normal extension of $S$. Then $N W_{0}=W_{0} T$ where $W_{0}$ is the one-one mapping which takes the same value as $W$ at each point. Thus, an operator $T$ is a quasi-affine transform of a subnormal 
operator if and only if there is a one-one mapping intertwining $T$ and a normal operator.

\section{Simple properties.}

PROPOSITION 1. If $T$ is a quasi-affine transform of a subnormal operator, then $T$ has the single-valued extension property.

Proof. Let $N$ be a normal operator, $W_{0}$ be a one-one map such that $N W_{0}=W_{0} T$. Suppose $g$ is a vector-valued analytic function defined on an open, set such that $(T-\lambda) g(\lambda) \equiv 0$. Then we have $(N-\lambda) W_{0} g(\lambda)=$ $W_{0}(T-\lambda) g(\lambda)=0$ for all $\lambda$. Since normal operators have the singlevalued extension property, $W_{0} g(\lambda)=0$ for all $\lambda$. Since $W_{0}$ is one-one, we have $g=0$.

Lemma 1. (See Colojoară and Foiaş [1] Proposition 3.8.) If $T$ is an operator on $\mathscr{H}$ with the single-valued extension property and $F$ is a closed set in $\mathbf{C}$ such that $\mathscr{X}_{T}(F)$ is closed, then we have $\sigma\left(T \mathscr{X}_{T}(F)\right) \subset F$. In particular, if $\mathscr{X}_{T}(F)=\mathscr{H}$, then $\sigma(T) \subset F$.

PROPOSITION 2. If $T$ is a quasi-affine transform of the subnormal operator $S$ and $N$ is the minimal normal extension of $S$, then $\sigma(N) \subset \sigma(S) \subset$ $\sigma(T)$.

Proof. That $\sigma(N) \subset \sigma(S)$ is well-known. Suppose $W: \mathscr{H} \rightarrow \mathscr{K}$ is a quasi-affinity such that $S W=W T$. Then $W \mathscr{H}=W \mathscr{X}_{T}(\sigma(T)) \subset$ $\mathscr{X}_{S}(\sigma(T))$. Since $W H$ is dense in $\mathscr{H}$ and $\mathscr{X}_{S}(\sigma(T))$ is closed (see Radjabalipour [7]), $\mathscr{X}_{S}(\sigma(T))=\mathscr{K}$. By the above lemma $\sigma(S) \subset \sigma(T)$.

REMARK 1. Using the same argument as above we can show that if $T$ is a quasi-affine transform of the hyponormal operator $S$, then $\sigma(S) \subset \sigma(T)$.

REMARK 2. Let $S$ be a subnormal operator on $\mathscr{H}$ and $N$ be the minimal normal extension of $S$ on $\mathscr{K}$. Then $S^{*} P=P N^{*}$, where $P$ is the projection from $\mathscr{K}$ onto $\mathscr{H}$. Therefore we have $\mathscr{H}=P \mathscr{K}=$ $P \mathscr{X}_{N^{*}}\left(\sigma\left(N^{*}\right)\right) \subset \mathscr{X}_{S^{*}}\left(\sigma\left(N^{*}\right)\right)$. If $S^{*}$ has the single-valued extension property, then, by Lemma $1, \sigma\left(S^{*}\right) \subset \sigma\left(N^{*}\right)$ and hence $\sigma(S)=\sigma(N)$.

ExAmPle. Let $S$ be the unilateral shift. Then its minimal normal extension is the bilateral shift, denoted by $U$. Note $\sigma(U)=$ the unit circle $\neq$ the unit disk $\approx \sigma(S)$. Hence, from the above remark, $S^{*}$ does 
not have the single-valued extension property. For a construction of a nonzero analytic function $g$ such that $\left(S^{*}-\lambda\right) g(\lambda) \equiv 0$, see Colojoară and Foiaş [1] p. 10.

It is well-known that a completely subnormal operator does not have a nontrivial invariant subspace on which the operator is normal. The same holds for operators which are quasi-affine transforms of completely subnormal operators.

Proposition 3. If $T$ is a quasi-affine transform of a completely subnormal operator $S$, then $T$ has no nontrivial invariant subspace $\mathcal{M}$ such that $T \mid \mathcal{M}$ is normal.

Proof. Let $W_{0}$ be a quasi-affinity and $S W_{0}=W_{0} T$. Suppose $\mathcal{M}$ is an invariant subspace of $T$ such that $T \mid \mathcal{M}$ is normal. Let $\mathcal{N}$ be the closure of $W_{0} \mathcal{M}$ and $W_{1}: \mathcal{M} \rightarrow \mathcal{N}$ be defined by restricting $W_{0}$ to $\mathcal{M}$. Then $\mathcal{N}$ is an invariant subspace of $S$ and hence $S \mid \mathcal{N}$ is subnormal. Also $(S \mid \mathcal{N}) W_{1}=W_{1}(T \mid \mathcal{M})$. Therefore $S \mid \mathcal{N}$ is normal. (See e.g. Radjavi and Rosenthal [8].) Since $S$ is subnormal, $\mathcal{N}$ is reducing for $S$. Since we assume that $S$ is completely subnormal, we have $\mathcal{N}=\{0\}$. Hence $\mathcal{M}=\{0\}$.

\section{Spectral manifolds.}

Proposition 4. If $T$ is an operator on $\mathscr{H}$ which is a quasi-affine transform of a subnormal operator $S, S^{*}$ has no point spectrum, $x \in \mathscr{H}, \Omega$ is an open set in $\mathrm{C}$ and $f: \Omega \rightarrow \mathscr{H}$ is a bounded function such that $(T-\lambda) f(\lambda)=x$ for all $\lambda$, then $f$ is analytic.

Proof. Let $N$ be the minimal normal extension for $S$ and $\mathscr{K}$ be the underlying Hilbert space of $N$. Let $W_{0}$ be a one-one mapping such that $N W_{0}=W_{0} T$. Since $S^{*}$ has no point spectrum, it is easy to show that $N$ also has no point spectrum. (From $N W_{0}=W_{0} T$ and the fact that $W_{0}$ is one-one we see that the point spectrum of $T$ is empty.) For $\lambda \in \Omega$, we have

$$
(N-\lambda) W_{0} f(\lambda)=W_{0}(T-\lambda) f(\lambda)=W_{0} x .
$$

By Putnam [5], $\lambda \rightarrow W_{0} f(\lambda)$ is analytic. Hence, for $y \in \mathscr{K}$, the function $\lambda \rightarrow\left(f(\lambda), W_{0}^{*} y\right)=\left(W_{0} f(\lambda), y\right)$ is analytic. Since $W_{0}$ is one-one, the range of $W_{0}{ }^{*}$ is dense and hence $\lambda \rightarrow(f(\lambda), x)$ is analytic for each $x$ in a dense subset of $\mathscr{H}$. By the boundedness of $f$, we can show that $\lambda \rightarrow(f(\lambda), x)$ is analytic for each $x$ in $\mathscr{H}$. Therefore $f$ is analytic.

For the next proposition we need a technical lemma. 
Lemma 2. Suppose that $\Omega$ is an open set in $\mathrm{C}, f: \Omega \rightarrow \mathscr{H}$ is a vector-valued function and $D$ is a dense subset of $\mathscr{H}$ such that $\lambda \rightarrow$ $(f(\lambda), x)$ is analytic for $x \in D$. Then there is an open dense subset $\Omega_{0}$ of $\Omega$ on which $f$ is analytic.

Proof. It suffices to show that, for every nonempty open subset $U$ of $\Omega$, there is a nonempty open subset of $U$ on which $f$ is bounded. Fix a nonempty open set $U$ in $\Omega$. First we show that, for every positive integer $n$, the set

$$
F_{n}=\{\lambda \in U:\|f(\lambda)\| \leqq n\}
$$

is relatively closed in $U$. Let $\lambda_{0} \in U$ be in the closure of $F_{n}$. Since, for $x \in D, \lambda \rightarrow(f(\lambda), x)$ is continuous and $|(f(\lambda), x)| \leqq n\|x\|$ for $\lambda \in F_{n}$, we have $\left|\left(f\left(\lambda_{0}\right), x\right)\right| \leqq n\|x\|$ for $x \in D$. Since $D$ is dense, $\left\|f\left(\lambda_{0}\right)\right\| \leqq$ $n$. Therefore $\lambda_{0} \in F_{n}$. Now, $U=\bigcup_{n=1}^{\infty} F_{n}$. By the Baire Category Theorem, there is some $n$ such that the interior of $F_{n}$ is nonempty. The proof is complete.

Proposition 5. If $T$ is an operator on $\mathscr{H}$ which is a quasi-affine transform of a subnormal operator $S, S^{*}$ has no point spectrum, $x \in \mathscr{H}, \Omega$ is an open set in $\mathbf{C}$ and $f: \Omega \rightarrow \mathscr{H}$ is a function such that $(T-\lambda) f(\lambda)=x$ for all $\lambda \in \Omega$, then there is a dense open subset $\Omega_{0}$ of $\Omega$ such that $f \mid \Omega_{0}$ is analytic.

Proof. The argument makes use of Lemma 2. It is a slight modification of that of Proposition 4, and hence is left to the reader.

COROLlARY. If $T$ on $\mathscr{H}$ is a quasi-affine transform of a subnormal operator $S$ on $\mathscr{K}, \Omega$ is a nonempty open subset of $\sigma(S)$ and $\cap\{(T-\lambda) \mathscr{H}: \lambda \in \Omega\} \neq\{0\}$, then $T$ has a nontrivial invariant subspace.

Proof. Suppose $S W=W T$ with $W$ as a quasi-affinity. If the point spectrum of $S^{*}$ is nonempty, from $W^{*} S^{*}=T^{*} W^{*}$ we see that the point spectrum of $T^{*}$ is also nonempty and hence $T$ has an invariant subspace. Therefore we may assume that the point spectrum of $S^{*}$ is empty. Let $x$ be a nonzero vector in $\cap\{(T-\lambda) \mathscr{H}: \lambda \in \Omega\}$. By Proposition 5 , there is a nonempty open set $\Omega_{0}$ in $\Omega$ such that $x \in$ $\mathscr{X}_{T}\left(\mathbf{C} \backslash \Omega_{0}\right)$. Let $\mathcal{M}$ be the closure of $\mathscr{X}_{T}\left(\mathbf{C} \backslash \Omega_{0}\right)$. Then $\mathcal{M} \neq\{0\}$. By Radjabalipour [7], $\mathscr{X}_{S}\left(\mathbf{C} \backslash \Omega_{0}\right)$ is closed. Since $\mathbf{C} \backslash \Omega_{0} \not \subset \sigma(S)$, by Lemma 1 , $\mathscr{X}_{S}\left(\mathbf{C} \backslash \Omega_{0}\right) \neq \mathscr{K}$. Now $W_{0} \mathscr{M} \subset \mathscr{X}_{S}\left(\mathbf{C} \backslash \Omega_{0}\right)$. Hence $\mathcal{M} \neq \mathscr{H}$.

Remark. In view of Stampfli and Wadhwa [12], Proposition 4 still 
holds if we merely assume that $T$ is a quasi-affine transform of a hyponormal operator without point spectrum.

4. Peak sets. The following theorem is a generalization of Theorem 1 in Putnam [6]:

THEOREM. Let $T$ (defined on $\mathscr{H}$ ) be a quasi-affine transform of a subnormal operator. Let $\Sigma$ be a spectral set of $T$ and $Q$ be a peak set of $R(\Sigma)$ (the uniform closure of rational function with poles off $\Sigma$ ). Then there is a projection $F(Q)$ on $\mathscr{H}$ such that $F(Q) \mathscr{H}=\mathscr{X}_{T}(Q)$ and $F(Q)$ is in the weakly closed inverse-closed algebra generated by $T$. Furthermore, $T \mid F(Q) \mathscr{H}$ and $T \mid(I-F(Q)) \mathscr{H}$ are quasi-affine transforms of subnormal operators and $Q$ is a spectral set for $T \mid F(Q) \mathscr{H}$.

Proof. Suppose $N=\int \lambda d E_{\lambda}$ on $\mathscr{K}_{0}$ is a normal operator, $W_{0}$ is a one-one mapping and $N W_{0}=W_{0} T$. Since $\Sigma$ is a spectral set of $T, g(T)$ is defined for $g \in R(\Sigma)$ and $\|g(T)\| \leqq \sup \{|g(\lambda)|: \lambda \in \Sigma\}$. Furthermore, it is straightforward to show that $g(N) W_{0}=W_{0} g(T)$ for $g \in R(\Sigma)$. Let $f$ be a peak function of $Q$, i.e., $f=1$ on $Q$ and $|f(\lambda)|<1$ for $\lambda \notin Q$. Then

$$
\left\|f(T)^{n}\right\| \leqq \sup \left\{\left|f(\lambda)^{n}\right|: \lambda \in \Sigma\right\} \leqq 1
$$

for each $n$. Hence $\left\{f(T)^{n}: n=1,2, \ldots\right\}$ has a weakly convergent subsequence, say, $w-\lim f(T)^{n_{\jmath}}=F(Q)$. Since $\left\{f^{n}: n=1,2, \ldots\right\}$ converges pointwisely to the characteristic function of $Q$ and $f(N)^{n} W_{0}=W_{0} f(T)^{n}$ for all $n$, we have $E(Q) W_{0}=W_{0} F(Q)$. Since $W$ is one-one and $W_{0} F(Q)^{2}=E(Q)^{2} W_{0}=E(Q) W_{0}=W_{0} F(Q)$, we have $F(Q)^{2}=F(Q)$. Since $\|F(Q)\| \leqq 1$, we see that $F(Q)$ is a projection. From the definition of $F(Q)$ we see that $F(Q)$ is in the weakly closed inverse-closed algebra generated by $T$.

For convenience, we write $T_{1}=T\left|F(Q) \mathscr{H}, N_{1}=T\right| E(Q) \mathscr{K}_{0}$ and $W_{1}: F(Q) \mathscr{H} \rightarrow E(Q) \mathscr{K}_{0}$ for the restriction of $W_{0}$ to $F(Q) \mathscr{H}$. We have $N_{1} W_{1}=W_{1} T_{1}$. Note that $W_{1}$ is one-one, $N_{1}$ is normal and $\sigma\left(N_{1}\right) \subset Q$.

Let $q$ be a rational function with poles off $\Sigma$. Let $C$ be an arbitrary compact set in $\mathbf{C}$ disjoint from $Q$. Then, when $n$ is large enough, we have

$$
\left\|q(T) f(T)^{n}\right\| \leqq \sup \left\{\left|q(\lambda) f(\lambda)^{n}\right|: \lambda \in \Sigma \mid C\right\} .
$$

Hence we have $\|q(T) F(Q)\| \leqq \sup \{|q(\lambda)|: \lambda \in \Sigma \mid C\}$. Since $C$ is arbitrary, we have 


$$
\left\|q\left(T_{1}\right)\right\|=\|q(T) F(Q)\| \leqq \sup \{|q(\lambda)|: \lambda \in Q\} .
$$

Next, suppose $r$ is a rational function with poles off $Q$. Since $Q$ is a peak set of $R(\Sigma)$, for every connected component $\Omega$ of $\mathbf{C} \backslash Q$, we have $\Omega \not \subset \Sigma$. (Otherwise, $f-1$ would be a nonzero continuous function which is analytic on $\Omega$ and zero on $\partial \Omega$, contradicting the maximal modulus principle.) By Rudin [10] Theorem 13.9, there is a sequence $\left\{q_{n}\right\}$ of rational functions with poles off $\Sigma$ such that $\sup \left\{\left|q_{n}(\lambda)-r(\lambda)\right|: \lambda \in Q\right\}$ $\rightarrow 0$ as $n \rightarrow \infty$. Hence, by $(*)$,

$$
\left\|q_{n}\left(T_{1}\right)-q_{m}\left(T_{1}\right)\right\| \leqq \sup \left\{\left|q_{n}(\lambda)-q_{m}(\lambda)\right|: \lambda \in Q\right\} \rightarrow 0
$$

as $n, m \rightarrow \infty$. Therefore $\left\{q_{n}\left(T_{1}\right): n=1,2, \ldots\right\}$ is convergent in the norm topology, to $T_{r}$, say. It is easy to see that $\left\|T_{r}\right\| \leqq \sup \{|r(\lambda)|: \lambda \in Q\}$, $r\left(N_{1}\right) W_{1}=W_{1} T_{r}$ and $T_{r}$ is in the inverse-closed, uniformly closed algebra generated by $T_{1}$. In particular, if $\mu \notin Q$ and $r$ is taken to be the function $\lambda \rightarrow(\lambda-\mu)^{-1}$, then $\left(N_{1}-\mu\right)^{-1} W_{1}=W_{1} T_{r}$ and

$$
W_{1}=\left(N_{1}-\mu\right)^{-1}\left(N_{1}-\mu\right) W_{1}=\left(N_{1}-\mu\right)^{-1} W_{1}\left(T_{1}-\mu\right)=W_{1} T_{r}\left(T_{1}-\mu\right) .
$$

Since $W_{1}$ is one-one, we have $T_{r}\left(T_{1}-\mu\right)=I$. Therefore $T_{1}-\mu$ is invertible. We have shown that $\sigma\left(T_{1}\right) \subset Q$. Now it is easy to see that, for general $r, T_{r}=r\left(T_{1}\right)$. Hence $Q$ is a spectral set for $T_{1}$.

Since $\sigma\left(T_{1}\right) \subset Q$, we have $F(Q) \mathscr{H} \subset \mathscr{X}_{T}(Q)$. Conversely, suppose $x \in \mathscr{X}_{T}(Q)$. Then there is an analytic vector-valued function $f: \mathbf{C} \backslash Q \rightarrow \mathscr{H}$ such that $(T-\lambda) f(\lambda)=x$ for all $\lambda$. Hence, for $\lambda \notin Q$, $(N-\lambda) W_{0} f(\lambda)=W_{0}(T-\lambda) f(\lambda)=W_{0} x$. Therefore $W_{0} x \in \mathscr{X}_{N}(Q)=$ $E(Q) \mathscr{K}_{0}$. Now $W_{0} F(Q) x=E(Q) W_{0} x=W_{0} x$. Since $W_{0}$ is one-one, $F(Q) x=x$, or $x \in F(Q) \mathscr{H}$. Therefore $F(Q) \mathscr{H}=\mathscr{X}_{T}(Q)$. The proof is complete.

REMARK 1. If we assume that $Q$, instead of being a spectral set for $T$, has the following property: there exists $M>0$ such that $\|r(T)\| \leqq$ $M \sup \{|r(\lambda)|: \lambda \in \Sigma\}$ for every rational function $r$ with poles off $\Sigma$, then, using the same argument as in the proof of the above theorem, we can establish the existence of an idempotent operator $F(Q)$ in the weakly closed, inverse-closed algebra generated by $T$ such that $F(Q) \mathscr{H}=$ $\mathscr{X}_{T}(Q)$. Furthermore, we have

$$
\|r(T \mid F(Q) \mathscr{H})\| \leqq M \sup \{|r(\lambda)|: \lambda \in Q\}
$$

for every rational function $r$ with poles off $Q$. Such an $F(Q)$ is unique. (Suppose $F_{1}$ and $F_{2}$ are two idempotent operators in the weakly 
closed, inverse-closed algebra generated by $T$ such that $F_{1} \mathscr{H}=F_{2} \mathscr{H}=$ $\mathscr{X}_{T}(Q)$. Then $F_{1} F_{2}=F_{2} F_{1}$ is also an idempotent operator with $F_{1} F_{2} \mathscr{H}=$ $F_{1} \mathscr{H}$ and $\operatorname{ker} F_{1} F_{2} \subset \operatorname{ker} F_{1}$. Hence $F_{1} F_{2}=F_{1}$. Similarly $F_{2} F_{1}=F_{1}$. Therefore $F_{1}=F_{2}$.)

REMARK 2. From the proof of $F(Q) \mathscr{H} \supset \mathscr{X}_{T}(Q)$ and in view of Putnam [5], we see that

$$
F(Q) \mathscr{H}=\mathscr{X}_{T}(Q)=\bigcap\{(T-\lambda) \mathscr{H}: \lambda \notin Q\} .
$$

REMARK 3. If $Q_{1}$ and $Q_{2}$ are peak sets for $\Sigma$, then we have $W_{0} F\left(Q_{1} \cap Q_{2}\right)=E\left(Q_{1} \cap Q_{2}\right) W_{0}=E\left(Q_{1}\right) E\left(Q_{2}\right) W_{0}=E\left(Q_{1}\right) W_{0} F\left(Q_{2}\right)$ $W_{0} F\left(Q_{1}\right) F\left(Q_{2}\right)$ and hence $F\left(Q_{1} \cap Q_{2}\right)=F\left(Q_{1}\right) F\left(Q_{2}\right)$. In general, let $\mathscr{B}$ be the Boolean algebra generated by the family of peak sets for $R(\Sigma)$. Then $F$ can be extended to $\mathscr{B}$ in a unique way such that:

(1) $F\left(B_{1} \cap B_{2}\right)=F\left(B_{1}\right) F\left(B_{2}\right)$

(2) $F\left(B_{1} \backslash B_{2}\right)=F\left(B_{1}\right)-F\left(B_{1}\right) F\left(B_{2}\right)$.

In fact, for $B_{1} \in \mathscr{B}, E\left(B_{1}\right) W_{0}=W_{0} F\left(B_{1}\right)$.

The following corollary is a generalization of a result in Conway and Olin [4].

COROLlARY. Let $T$ be a completely nonnormal contraction which is a quasi-affine transform of a subnormal operator with minimal normal extension $N=\int \lambda d E_{\lambda}$ on $\mathscr{K}_{0}$. If $Z$ is a Borel set in $\{\lambda \in \mathbf{C}:|\lambda|=1\}$ of arc length measure zero, then $E(Z)=0$.

Proof. By the inner regularity of the spectral measure $E$, it suffices to prove the corollary under the additional assumption that $Z$ is closed. Since $T$ is a contradiction, by von Neumann's well-known theorem, the closed unit disc $\Sigma=\{\lambda:|\lambda| \leqq 1\}$ is a spectral set for $T$. By the theorem of F. and M. Riesz (see, e.g., Hoffman [2], p. 32), $Z$ is a peak set for $R(\Sigma)$. From the above theorem, we have $E(Z) W_{0}=W_{0} F(Z)$ $\left(W_{0}: \mathscr{H} \rightarrow \mathscr{K}_{0}\right.$ here is a one-one mapping implementing $N W_{0}=W_{0} T$ ), and $Z$ is a spectral set for $T \mid F(Z) \mathscr{H}$. By the Hartogs-Rosenthal Theorem, $R(Z)=C(Z)$. Therefore $T \mid F(Z) \mathscr{H}$ is normal, (by Lebow [3]). Since, by assumption, $T$ is completely nonnormal, $F(Z)=$ 0 . Hence $E(Z) W_{0}=0$. Since $N$ is the minimal normal extension of the subnormal operator given by restricting $N$ to the closure of the range 
of $W_{0}, \mathscr{K}_{0}$ is the closure of the linear span of $\left\{N^{* n} x: x \in W_{0} \mathscr{H}, n=\right.$ $1,2, \cdots\}$. Therefore $E(Z)=0$.

\section{REFERENCES}

1. I. Colojoară and C. Foiaş, Theorey of Generalized Spectral Operators, Gordan and Breach, New York, 1968.

2. K. Hoffman, Banach Spaces of Analytic Functions, Prentice-Hall, Englewood Cliffs, New Jersey, 1962.

3. A. Lebow, On von Neumann's theory of spectral sets, J. Math. Anal. Appl., 7 (1963), 64-90.

4. J. B. Conway and R.F. Olin, A functional calculus for subnormal operators, II, (to appear).

5. C.R. Putnam, Ranges of normal and subnormal operators, Michigan Math. J., 18 (1971), 33-36.

6. — Peak sets and subnormal operators, (to appear).

7. M. Radjabalipour, Ranges of hyponormal operators, Illinois J. Math., (to appear).

8. H. Radjavi and P. Rosenthal, On roots of normal operators, Math. Anal. \& Appl. 34 (1971), 653-664.

9. H. Radjavi and P. Rosenthal, Invariant Subspaces, Springer-Verlag, New York, 1973.

10. W. Rudin, Real and Complex Analysis, McGraw-Hill, New York, 1966.

11. J.G. Stampfli and B.L. Wadhwa, An asymmetric Putnam-Fuglede theorem for hyponormal operators, Indiana U. Math. J., 25 (1976), 359-365.

12. J.G. Stampfli and B.L. Wadhwa, On dominant operators, (to appear).

Received September 14, 1976 and in revised form September 14, 1977. This work was partially supported by the National Science Foundation.

UNIVERSITY OF TORONTO

TORONTO M5S - 1A1

ONTARIO, CANADA 


\section{PACIFIC JOURNAL OF MATHEMATICS \\ EDITORS}

RICHARD ARENS (Managing Editor)

University of California

Los Angeles, CA 90024

\section{R. A. Beaumont \\ University of Washington \\ Seattle, WA 98105}

C. C. MOORE

University of California

Berkeley, CA 94720

\section{J. DUGUNDJI}

Department of Mathematics

University of Southern California

Los Angeles, CA 90007

R. FINN AND J. MILGRAM

Stanford University

Stanford, CA 94305

\section{ASSOCIATE EDITORS}

\section{E. F. BECKENBACH}

B. H. NeUmanN

F. WOLF

K. YoSHIDA

\section{SUPPORTING INSTITUTIONS}

\author{
UNIVERSITY OF BRITISH COLUMBIA \\ CALIFORNIA INSTITUTE OF TECHNOLOGY \\ UNIVERSITY OF CALIFORNIA \\ MONTANA STATE UNIVERSITY \\ UNIVERSITY OF NEVADA \\ NEW MEXICO STATE UNIVERSITY \\ OREGON STATE UNIVERSITY \\ UNIVERSITY OF OREGON \\ OSAKA UNIVERSITY
}

\author{
UNIVERSITY OF SOUTHERN CALIFORNIA \\ STANFORD UNIVERSITY \\ UNIVERSITY OF HAWAII \\ UNIVERSITY OF TOKYO \\ UNIVERSITY OF UTAH \\ WASHINGTON STATE UNIVERSITY \\ UNIVERSITY OF WASHINGTON \\ AMERICAN MATHEMATICAL SOCIETY
}

The Supporting Institutions listed above contribute to the cost of publication of this Journal, but they are not owners or publishers and have no responsibility for its contents or policies.

Mathematical papers intended for publication in the Pacific Journal of Mathematics should be in typed form or offset-reproduced (not dittoed), double spaced with large margins. Underline Greek letters in red, German in green, and script in blue. The first paragraph or two must be capable of being used separately as a synopsis of the entire paper. Items of the bibliography should not be cited there unless absolutely necessary, in which case they must be identified by author and Journal, rather than by item number. Manuscripts, in duplicate, may be sent to any one of the four editors. Please classify according to the scheme of Math. Reviews, Index to Vol. 39. All other communications should be addressed to the managing editor, or Elaine Barth, University of California, Los Angeles, California, 90024.

100 reprints are provided free for each article, only if page charges have been substantially paid. Additional copies may be obtained at cost in multiples of 50 .

The Pacific Journal of Mathematics is issued monthly as of January 1966. Regular subscription rate: $\$ 72.00$ a year (6 Vols., 12 issues). Special rate: $\$ 36.00$ a year to individual members of supporting institutions.

Subscriptions, orders for back numbers, and changes of address should be sent to Pacific Journal of Mathematics, 103 Highland Boulevard, Berkeley, California, 94708.

PUBLISHED BY PACIFIC JOURNAL OF MATHEMATICS, A NON-PROFIT CORPORATION

Printed at Jerusalem Academic Press, POB 2390, Jerusalem, Israel.

Copyright (C) 1977 Pacific Journal of Mathematics All Rights Reserved 


\section{Pacific Journal of Mathematics \\ Vol. 70, No. $2 \quad$ October, 1977}

B. Arazi, A generalization of the Chinese remainder theorem ........... 289

Thomas E. Armstrong, Polyhedrality of infinite dimensional cubes .... . . . 297

Yoav Benyamini, Mary Ellen Rudin and Michael L. Wage, Continuous

images of weakly compact subsets of Banach spaces ............ 309

John Thomas Burns, Curvature functions on Lorentz 2-manifolds ......... 325

Dennis F. De Riggi and Nelson Groh Markley, Shear distality and equicontinuity .................................. 337

Claes Fernström, Rational approximation and the growth of analytic

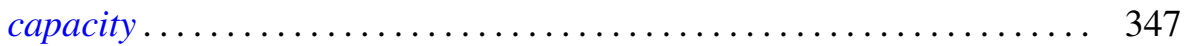

Pál Fischer, On some new generalizations of Shannon's inequality....... 351

Che-Kao Fong, Quasi-affine transforms of subnormal operators ......... 361

Stanley P. Gudder and W. Scruggs, Unbounded representations of

*-algebras........................................ 369

Chen F. King, A note on Drazin inverses .................... 383

Ronald Fred Levy, Countable spaces without points of first countability . . . 391

Eva Lowen-Colebunders, Completeness properties for convergence

spaces ......................................... 401

Calvin Cooper Moore, Square integrable primary representations ....... 413

Stanisław G. Mrówka and Jung-Hsien Tsai, On preservation of

E-compactness ................................ 429

Yoshiomi Nakagami, Essential spectrum $\Gamma(\beta)$ of a dual action on a von

Neumann algebra ................................ 437

L. Alayne Parson, Normal congruence subgroups of the Hecke groups

$G\left(2^{(1 / 2)}\right)$ and $G\left(3^{(1 / 2)}\right)$...

Louis Jackson Ratliff, Jr., On the prime divisors of zero in form rings . . . . 489

Caroline Series, Ergodic actions of product groups .................. 519

Robert O. Stanton, Infinite decomposition bases..................... 549

David A. Stegenga, Sums of invariant subspaces .................. 567 